\title{
A Survey of Weather Forecasting based on Machine Learning and Deep Learning Techniques
}

\author{
Mihir Bhawsar ${ }^{1}$, Vandan Tewari ${ }^{2}$, Preeti Khare ${ }^{3}$ \\ ${ }^{1}$ Computer Science Engineering, SGSITS Indore, India, \\ Email: mihirbhawsar143@gmail.com \\ ${ }^{2}$ Computer Science Engineering, SGSITS Indore, India, \\ Email: vandantewari@gmail.com \\ ${ }^{3}$ Computer Science Engineering, SGSITS Indore, India, \\ Email: preetikhr4@gmail.com
}

\begin{abstract}
Forecast of future weather is one of the dominant concerns in today's era. Several techniques that have been used to forecast future weather are statistical analysis, machine learning, and deep learning techniques. Forecast of weather can assist in the decision-making process for the prevention of disasters. While forecasting weekly weather can help people, decide and manage their activities and recreational programs it can also help Smart management programs. The accuracy of the Short term ambient forecast conditions is particularly conducive to the development of forecast management strategies. This paper presents an inclusive survey on such methods based on Machine Learning, and Deep Learning Techniques used to predict weather and discusses the applications, types, and problems of the forecasting.
\end{abstract}

Key words: Weather Forecast, Machine Learning, Deep Learning Techniques, Data Mining

\section{INTRODUCTION}

Forecasting has pulled in the consideration of numerous researchers due to its impact on the health of humans. The availability of large-scale forecasting data and the origin of computer information and technology over the past decade have encouraged researchers to explore mysterious patterns in large-scale weather forecasting databases. Forecasting of Weather is a research project with a wide range of potential applications from air travel to agriculture and tourism. Forecasting is a complex and challenging skill that involves observing and processing a large amount of data.

Over the past decade, many important efforts to predict the weather using mathematical modeling techniques including machine learning, deep learning, data mining have been done. Methods include persistence, weather, viewing the sky, barometer use, nowcasting, use of predictive models, analog and predict together. Predictability can be used by air traffic, weather warnings, maritime, agricultural, utility companies, private sector, military. Forecasting is a scientific challenge based on good weather monitoring, data analysis by meteorologists and computers, and a fast communication system.
Weather forecasting is done using relevant details about the present status of the atmosphere and utilizing a scientific understanding of the cosmic technique to show how the universe will change in the next few hours.

There are many different end-to-end user predictions. Weather alerts are important predictions because they are used for the protection of health and property. Predictions based on temperature and rainfall are important in agriculture, and therefore in order traders within the stock market. Temperature forecasts are also used by companies to measure more demand days to come. Every day people use the weather forecast to find out what to wear on a given day.

\section{RELATED WORK}

We inspected a few papers on machine learning for the prediction of weather, Mark Holmstrom, Dylan Liu, Christopher Vo [1] investigate their application to climate determining to conceivably produce more exact climate gauges for huge time frames. On the other hand, Sanjay D. Sawaitul, Prof. K. P. Wagh, Dr. P. N. Chatur [2] discussed different models which were used in the past for weather forecasting. Fahad Sheikh, S. Karthick, D. Malathi, J. S. Sudarsan, and C. Arun [3] has discussed C4.5 and Na"1ve Bayes algorithm, a novel strategy for foreseeing the kinds of weather dependent on the PV power data and partial meteorological data was discussed by Wenying Zhang, Huaguang Zhang, Fellow, IEEE, Jinhai Liu, Kai Li, Dongsheng Yang, and Hui Tian [4]. G.Vamsi Krishna [5] using the autoregressive integrated moving average (ARIMA) model to estimate the future worth. In this model, at first, a numerical model is produced by thinking about an ordered group of data, and afterward, the forecast is completed by using the model utilizing the current qualities and the past information. Autocorrelation models and partial autocorrelation models are likewise considered for playing out the interventional investigation. Janani. B, Priyanka Sebastian [6] focus on different procedures that are utilized for weather prediction. R. Samya, R. Rathipriya, [7] review the different forecast procedures for cloudburst utilizing Data Mining and Artificial Neural Network (ANN). The survey of Data Mining Techniques for Weather Prediction and studies the advantage of utilizing it was discussed by Divya Chauhan, Jawahar Thakur [8]. Divya Chauhan, Jawahar Thakur [8] additionally give a review of 
Mihir Bhawsar et al., International Journal of Emerging Trends in Engineering Research, 9(7), July 2021, 988 - 993

the accessible writing of certain algorithms utilized by various researchers to use different data mining methods, for Weather Prediction. Prashant Biradar, Sarfraz Ansari, YashavantParadkar, Savita Lohiya [9] talked about the utilization of K-medoids and Naive Bayes algorithm for weather determining framework with boundaries like temperature, moistness, and the breeze. In this way, successful models to predict weather are to be analyzed. One paper constructs effective models to predict the weather. Neural networks and gradient boosting classifier. S. Nagadevi, Varun Ramesh, Helloween James are using four different machine learning algorithms for predicting weather. Algorithms - KNearestNeighbour, Decision Tree, Random Forest Algorithm, Linear Regression.

\section{TYPES OF WEATHER FORECASTING}

\subsection{CLIMATOLOGY METHOD}

This method provides a process to generate a weather forecast. After reviewing weather data collected over many years and calculating estimates Meteorologists use this method.

\subsection{ANALOG METHOD}

This method can be used when forecasting the weather because it requires finding the date in the past with weather it's like a current forecast, which is hard to do.

\subsection{PERSISTENCE AND TRENDS METHOD}

Persistence and inclination require less expertise to predict the weather because it depends on past trends. In the world, the weather is changing, much like the weather forecast for today. This only requires that you stay up-to-date with current temperatures and know the weather conditions in the region.

\subsection{NUMERICAL WEATHER PREDICTION}

Weather forecasts on prices depend on computers for finding the weather. Larger supercomputers help meteorologists to make weather forecasts based on many weather conditions.

\subsection{LOOKING AT THE SKY}

By seeing at the sky you can determine weather by seeing the sun or the moon or if you are looking at very high clouds. In the event that the mists are cloudy, there is an opportunity for an awful climate in a little while later on. To decide when the tempest is coming, look at whether the clouds are moving or not.

\subsection{USE OF A BAROMETER}

Barometric pressure has been used in predictions since the 19th century. At the point when a low-pressure framework is drawing closer, and there is a more noteworthy possibility of a downpour. Pressure increments are related to improved weather conditions.

\subsection{NOWCASTING}

Weather forecasts for the next six hours are commonly called nowcasting. At this point, it is possible to predict small things like individual thunderstorms with the right precision, just as different things that are too little to ever be addressed by a computer.

\subsection{USE OF FORECASTING MODELS}

Human forecasters were answerable for creating all the weather as indicated by available data. Today, human info is frequently confined to model selection dependent on different boundaries, e.g. model discrimination and performance. It uses the harmony of climate models, as well as the integration of members of a wide range of species, can help decrease environmental mistakes. In any case, regardless of how minor the mistake may be in any individual system, major errors within any specific directional correction may occur in any given model operation. Individuals can utilize data about local results, which might be too little in size to be settled by a model for adding details to a forecast.

\subsection{ENSEMBLE FORECASTING}

Although the weather model will predict changing weather conditions for the distant future, errors in the forecast will inevitably grow over time due to atmospheric turmoil and inconsistencies at first sight. The information that can be given in the forecast diminishes after some time as these mistakes increment. This turns into a point where the mistakes are incredible to such an extent that the estimate can't meet the genuine atmosphere.

However, looking at one prediction offers there is no indication of the probability of such a prediction accuracy. Predictability includes the production of multiple predictions in sequence to show uncertainty at first atmosphere (due to errors visual and inadequate sample). Uncertainty is a prediction that can be checked for various predictions are generated.

Ensemble forecasts continue to be used for weather forecasts Group expectation requires a perplexing comprehension of the universe and computer models.

\section{UTILIZATION'S OF WEATHER FORECASTING}

Predictions of the future weather can be applied to the following areas:

\subsection{TOUGH WEATHER WARNINGS AND ADVICE}

In today's weather forecasts there are weather warnings and advice, issued by national meteorologists in a situation where bad or dangerous weather is normal. This warning is to secure life of humans and their belongings. Some of the 
Mihir Bhawsar et al., International Journal of Emerging Trends in Engineering Research, 9(7), July 2021, 988 - 993

worst weather forecasts are known as hurricanes and hurricane warnings, as well as the latest flood warnings in parts of Nigeria by the National Meteorological Agency. Different kinds of exhortation incorporate winter weather, strong winds, floods, cyclones, and fog. Extensive advice and warnings are broadcast through the media, including radio, utilizing emergency services, for example, the Emergency Alert System, which incorporates into the standard system.

\subsection{AIR TRAFFIC}

The navigation industry is highly touchy to the climate, careful climate estimates are important because the majority of recorded plane crashes worldwide have weather-related causes.

\subsection{MARINE}

The utilization of waterways can be severely restricted by wind and speed, earthquake time, high waves, and rain. These may contribute to the safety of navigation. As a result, different types of codes were developed to accurately convey detailed maritime weather forecasts to send pilots by radio.

\subsection{AGRICULTURE}

Ranchers rely upon climate estimates to sort out what work they will do on some random day. Dry seasons can harm crops. While plants can be harmed by the dry season, their dried buildups can be used as feed for steers as silage. Snow and cold play ruin with plants both spring and harvest time. For instance, a completely developed peach tree can make their peach crop free from frostbite. Orange groves can be severely damaged during snow and cold, regardless of the season.

\subsection{COMPANIES USED}

Electricity and gas organizations rely upon weather estimates to satisfy requests, which could be essentially influenced by the weather. They use a value called degree-day to determine how much energy will be used in heat or cooling. These qualities depend on the everyday temperature of between $650 \mathrm{~F}$ (180C). Cool temperatures force the hot days, while hotter temperatures power cooling days. In winter, a freezing climate can make an expansion sought after as individuals raise their temperature. Essentially, in the summer or during the dry season an expansion popular might be related to expanded utilization of cooling frameworks in sweltering climate. In anticipation of an increase in demand, service providers may produce a power supply or natural gas before the value rises, or in some cases, goods are limited by the utilization of black spots and power outages.

\subsection{PRIVATE SECTOR}

Privately owned businesses are paying for weather forecasts to discover their requirements to build their benefits and stay away from significant misfortunes. Climate estimates can be utilized to put resources into the stock market, like the eventual fate of oranges, corn, soybeans, and oil. Additionally, community members utilize future climate data to choose what to wear, every day.

\subsection{MILITARY APPLICATIONS}

Military weather forecasts are weather conditions including war, society.

Military weather forecasts give pre-appearance climate data to pilots and give genuine security insurance services conveyed in the military.

\section{MACHINE LEARNING TECHNIQUES USED IN WEATHER FORECASTING}

So far, anything we have seen is traditional weather forecasts. These methods are not stable and tend to make mistakes in finding. In addition, we cannot predict big time to use them because they become inaccurate at the time. The Most commonly utilized machine learning techniques for weather forecasting are Naive Bayes, Decision Tree, Neural Networks, Rule-based Methods, Support Vector Machine, Bayesian Belief Network, KNearest Neighbor, Random Forest Algorithm, Linear Regression. There are usually only two machine learning methods used to predict the weather i.e. Bayesian Network and Neural Network.

\subsection{NEURAL NETWORK}

Neural Networks are extremely amazing in nature so it is the most famous approach to contemplate weather forecasting equipment. The primary justification for this notoriety is the capacity to catch non-linear conditions of the past and future weather conditions, in contrast to the reversal of the line and models of active order [12].

\subsection{BAYESIAN NETWORK}

Bayesian Network utilizes machine learning algorithms to decide the right Bayesian Network and boundaries [temperature, stickiness, see, etc.]. The expense of the Bayesian Network computation is extravagant because of an enormous number of various conditions [18].

\section{MACHINE LEARNING SEQUENCE IN DATA MINING TECHNIQUES}

\subsection{DATA COLLECTION}

The information utilized was gathered at the meteorologist's center. The accompanying advances were adopted in this exploration stage: Data Cleaning, Data Selection, Data Transformation, and Data Mining. 
Mihir Bhawsar et al., International Journal of Emerging Trends in Engineering Research, 9(7), July 2021, 988 - 993

\subsection{DATA CLEANING}

At this stage, the corresponding data model format was created which is to look for missing information, recover information, and erase awful information. Eventually, the system cleanup details were converted into the appropriate data mining format.

\subsection{DATA SELECTION}

Data relating to analysis as a decision tree was cut and extracted from the database.

\subsection{DATA TRANSFORMATION}

"This is also known as data consolidation". It is the stage wherein the picked data is changed into structures appropriate for Data mining. The data file was saved in Commas Separated Value (CVS) document format and the dataset was normalized to diminish the effect of scaling on the data.

\subsection{DATA MINING STAGE}

The data mining phase is separated into three stages. In each stage, all algorithms are utilized to analyze the climate data set. The test method adopted in this study is to divide the train training percentage into the data percentage, cross it working on it and test the leftover rates. From there on the revelation of interesting patterns represent information.

\section{DATA MINING TECHNIQUES USED IN WEATHER FORECASTING}

The way toward getting fascinating information from a gigantic data known as Data Mining. Data sources are databases, data warehouses, the web, and other information repositories. Furthermore, it is iterative sequence data. The most utilized Data mining procedure are classification, clustering, Decision Trees.

\subsection{CLASSIFICATION}

Classification means tracking down a model that classifies data classes for having the option to utilize a model to foresee the class of objects with their unknown class label [11]. Different classification algorithms are Backpropagation, KNN and, genetic algorithm.

\subsection{CLUSTERING}

Clustering investigations data objects without contact with a notable category label. The unsupervised learning method of the clustering process is a helpful method to discover styles and patterns in data, where there are no pre-defined classes [11]. Some of the clustering algorithms are K- mean and k- media.

\subsection{DECISION TREE}

Structural structures such as trees representing decision sets. These decisions generate data classification rules [14].

\section{DEEP LEARNING TECHNIQUES FOR WEATHER FORECASTING}

Up until this point, anything we have seen is known as conventional weather forecasts. These conventional techniques are flimsy and often make prediction mistakes. Moreover, we cannot predict a great time to use them because they become inaccurate at the time. There are usually some deep learning methods used to predict the weather i.e. Convolution Network $(\mathrm{CN})$, Conditional Restricted Boltzmann Machines (CRBM), Recurrent Neural Network.

\subsection{RECURRENT NEURAL NETWORK}

Recurrent Neural Network (RNN) [19] is an artificial NN [17] used to anticipate a progression of times. Elman networks that are important for the RNN comprising of at least one secret layer. The primary layer has the weight found in the installation all the layers will get the load from the previous layer. This network has the function of activating which can be in the form of any function going on and on. Postpones that happened in the primary secret layer previously ( $t-l)$ can be applied to the present $(t)$. In contrast to the repetitive neural network is a feedback loop that conveys the details of the interruption (sound) of the previous input to be inserted into the next input.

\subsection{CONDITIONAL RESTRICTED BOLTZMANN MACHINES (CRBM)}

Restricted Boltzmann Machines are deep learning models to tackle different issues, for example, collaborative filtering, classification, and modelling motion capture data problems [19].

\subsection{CONVOLUTION NETWORK (CN)}

Convolutional network (CN) models [19], as well as neocognitrons [18], [19] known as one of the biologically inspired models.

LeCunet al. describes the deep $\mathrm{CN}$ structure can have several stages. For $\mathrm{CN}$ application we use color images as input, the $\mathrm{CN}$ commonly used in the background on the feature map can be a 2D list containing the color path of the input image (for audio input map for each feature could be an ID list, and for a video or a volumetric image, it could be a 3D list.

The common unsupervised training method for $\mathrm{CNN}$ to learn the filter coefficients in the filter bank layers is called Predictive Sparse Decomposition Algorithm [15]. 
Mihir Bhawsar et al., International Journal of Emerging Trends in Engineering Research, 9(7), July 2021, 988 - 993

\section{PROBLEMS OF WEATHER FORECASTING}

An objective of all scientific endeavors is to make exact forecasts. Physicists or chemists conducting laboratory tests do so in the desire for tracking down some fundamental rules that can be utilized to foresee the result of different tests dependent on those standards. Indeed, most laws of science are too precise expectations in regards to the result of particular sorts of tests. In any case, hardly a few physiologists are more worried about more complex or challenging predictors than a meteorologist.

\subsection{INCOMPLETE DATA}

Data for modern numerical models still contributes significantly to radiosonde imaging. However, the numerous radio broadcasts the World over has declined in the course of recent many years. The most evolved nations on the planet today, go through more cash to dispatch satellites than exhausting weather balloons. Satellite information is internationally normal, yet information coordination specialists are as yet attempting to sort out how this information can be" appropriately processed" by models. In addition, important climatic factors remain elusive, especially at sea. Model results are as good as the data in its original form.

\subsection{MISCONCEPTIONS "VISION" AND "FUTURES"}

Today's forecasts include the inevitable trade between horizontal and long-term resolutions. This is because good adjustment means more points than can be calculated. This requires a lot of computer time. The future forecast also requires counting millions or billions. When optimized with a long-range forecast, this task can squeeze the fastest supercomputers today. One would not get predictions for weeks. Future improvements to computers will help speed things up. So far, however, some species have not been able to select or "see" such small objects as clouds, raindrops, and snowflakes. To compensate for this complex "idea" of models, the computer code incorporates unlimited limitations into the unseen. These are called parameterizations. Even though science is deeply rooted in them, this approach does not come close to discovering the complex truth of the matter. This is because; the events of the smallest scale are often very frightening to understand.

Therefore, it is not an insult to the skills of astronomers to say that the performance of parameters is the "backbone" of reality.

\subsection{CONFLICT}

It is shocking to realize that, even if a large computer with 4,000 statistics per second were not established, no better predictable results would be obtained. The weather forecast for the best solution has its limitations.

The reason for these limitations is the ambitious asset of complex systems, which change like air. It is called "Empathy dependence on original conditions", and is a symbol of what is widely known as revolutionary theory. Space disorder doesn't imply that everything is demolished; all things being equal, it implies that the climate, in actuality, and the computer model can gain uniquely in contrast to the primary marginally various conditions.

\section{CONCLUSION}

Weather forecasting estimating is the usage of science and development to anticipate the states of the environment for a given area and time. Peoples have attempted to anticipate the climate casually for a significant long time and officially since the nineteenth century. Climate guesses are made by social occasion quantitative data about the current status of the environment at a given spot and utilizing meteorology to project how the climate will change.

With scientific advancements, a lot of research is going on weather gauging utilizing Data mining, deep learning, machine learning have been done. However, there was a lack of surveys available on the present status of exploration and application. This paper thus offers a survey of weather forecasting using various techniques. Also summarizing the key concepts and focusing on the existing work on weather forecasting, its types, and its applications. To conclude, how deep learning, data mining, and machine learning algorithms were employed in weather forecasting is exceptionally important to guarantee future exploration will focus destined for success, accordingly improving the performance of weather predictions.

\section{REFERENCES}

[1] Mark Holmstrom, Dylan Liu, Christopher Vo, Machine Learning Applied to Weather Forecasting. Stanford University (Dated: December 15, 2016)

[2] Sanjay D. Sawaitul, Prof. K. P. Wagh, Dr. P. N. Chatur, Classification and Prediction of Future Weather by using Back Propagation Algorithm-An

Approach. International Journal of Emerging Technology and Advanced Engineering (ISSN 2250-2459, Volume 2, Issue 1, January 2012

[3] Fahad Sheikh, S. Karthick, D. Malathi, J. S. Sudarsan and C. Arun, Analysis of Data Mining Techniques for Weather Prediction. Indian Journal of Science and Technology, Vol 9(38), DOI: 10.17485/ijst/2016/v9i38/101962, October 2016.

[4] Wenying Zhang, Huaguang Zhang, Fellow, IEEE, Jinhai Liu, Kai Li, Dongsheng Yang, and Hui Tian, Weather Prediction With Multiclass Support Vector Machines in the Fault Detection of Photovoltaic System. IEEE/CAA JOURNAL OF AUTOMATICA SINIC, VOL. 4, NO. 3, JULY 2017

[5] G.Vamsi Krishna, An Integrated Approach for Weather Forecasting based on Data Mining and Forecasting Analysis. 
Mihir Bhawsar et al., International Journal of Emerging Trends in Engineering Research, 9(7), July 2021, 988 - 993

International Journal of Computer Applications (0975 8887), Volume 120 No.11, June 2015

[6] Janani.B, Priyanka Sebastian, ANALYSIS ON THE WEATHER FORE-CASTING AND TECHNIQUES. International Journal of Advanced Research in Computer Engineering and Technology (IJARCET), Volume 3, Issue 1, January 2014

[7] R. Samya, R. Rathipriya, Predictive Analysis for Weather Prediction using Data Mining with ANN: A Study. International Journal of Computational Intelligence and Informatics, Vol. 6: No. 2, September 2016

[8] Divya Chauhan, Jawahar Thakur, Data Mining Techniques for Weather Prediction: A Review. Data Mining Techniques for Weather Prediction: A Review, Volume: 2 Issue: 8

[9] Prashant Biradar, Sarfraz Ansari, YashavantParadkar, Savita Lohiya, Weather Prediction Using Data Mining. 2017 IJEDR — Volume 5, Issue 2 - ISSN: 2321-9939

[10] Arpit Tiwari, S. K. Verma, Cloudburst Predermination System, IOSR Journal Of computer Engineering, Vol.17, No.3, pp 47-56, 2015.

[11] BadhiyeS.S ,Wakode B. V, Chatur P. N., Analysis Of Temperature And Humidity Data For Future Value Prediction, International Journal Of Computer Science And Information Technologies , Vol.3, pp.30123014, 2012.

[12] Rupa, A Review Study Of Rainfall Prediction Using Neuro-Fuzzy Inference System, International Journal Of Engineering Science and Research Technology, 2015.

[13] P.Kalaiyarasi, Mrs.A.Kalaiselvi, Data Mining Techniques Using To Weather Prediction, International Journal of Computer Science Trends and Technology (IJCST), May - June 2018

[14] C. Kunjumon, S. S. Nair, D. Rajan S., P. Suresh and S. L. Preetha, "Survey on Weather Forecasting Using Data Mining," 2018 Conference on Emerging Devices and Smart Systems (ICEDSS), Tiruchengode, India, 2018, pp. 262-264, doi: 10.1109/ICEDSS.2018.8544326.

[15] L. Han, J. Sun and W. Zhang, "Convolutional Neural Network for Convective Storm Nowcasting Using 3-D Doppler Weather Radar Data," in IEEE Transactions on Geoscience and Remote Sensing, vol. 58, no. 2, pp. 1487-1495, Feb. 2020, doi: 10.1109/TGRS.2019.2948070.

[16] P. Zhang, Y. Jia, J. Gao, W. Song and H. Leung, "ShortTerm Rainfall Forecasting Using Multi-Layer Perceptron," in IEEE Transactions on Big Data, vol. 6, no. 1, pp. 93-106, 1 March 2020, doi: 10.1109/TBDATA.2018.2871151.

[17] K. Yonekura, H. Hattori and T. Suzuki, "Short-term local weather forecast using dense weather station by deep neural network," 2018 IEEE International Conference on Big Data (Big Data), Seattle, WA, USA, 2018, pp. 1683-1690, doi: 10.1109/BigData.2018.8622195.

[18] V. B. Nikam and B. B. Meshram, "Modeling Rainfall Prediction Using Data Mining Method: A Bayesian Approach," 2013 Fifth International Conference on Computational Intelligence, Modelling and Simulation,
Seoul, Korea (South), 2013, pp. 132-136, doi: 10.1109/CIMSim.2013.29.

[19] A. G. Salman, B. Kanigoro and Y. Heryadi, "Weather forecasting using deep learning techniques," 2015 International Conference on Advanced Computer Science and Information Systems (ICACSIS), Depok, Indonesia, 2015, pp. 281-285, doi: 10.1109/ICACSIS.2015.7415154.

[20] Kolla, Bhanu \& Naga Pawan, Y V R \& Kalyani, Lakshmi \&Vadla, Pradeep. (2020). Chatterbot implementation using Transfer Learning and LSTM Encoder-Decoder Architecture (International Journal of Emerging Trends in Engineering Research ISSN: 2347 3983). 8. 1709-1715. 